\title{
Diagnostic value of endoscopic acetic acid staining combined with narrow band imaging in the diagnosis of intestinal metaplasia in gastric mucosa
}

\author{
Meili Gao, Ji Chen, Hong Cui \\ Department of Gastroenterology, The Third Affiliated Hospital of Inner Mongolia Medical University, Baotou, Inner Mongolia, \\ China
}

Received: January 5, 2018

DOI: $10.14725 /$ dcc.v5n1p5

Accepted: February 13, $2018 \quad$ Online Published: March 10, 2018

URL: http://dx.doi.org/10.14725/dcc.v5n1p5

\begin{abstract}
Early detection and diagnosis of intestinal metaplasia in gastric mucosa will be of great significance to delay or reverse the occurrence of gastric carcinoma. Acetic acid staining combined with narrow band imaging (NBI) was applied to this study, to investigate its value in the diagnosis of intestinal metaplasia in gastric mucosa.
\end{abstract}

Key Words: Intestinal metaplasia, Narrow band imaging, Gastric mucosa

Intestinal metaplasia in gastric mucosa refers to the replacement of gastric epitheliums by intestinal glandular epitheliums. Studies have suggested that intestinal metaplasia is closely related to the occurrence of gastric carcinoma. ${ }^{[1]}$ Therefore, early detection and diagnosis of intestinal metaplasia in gastric mucosa will be of great significance to delay or reverse the occurrence of gastric carcinoma. At present, gastroscopy combined with biopsy is considered as a good method to diagnose intestinal metaplasia in gastric mucosa, which is focally distributed mostly. It is easily misdiagnosed by means of random biopsy. Narrow band imaging (NBI) is a new endoscopic technique that can be used to observe the microstructure of the gastrointestinal mucosa and morphological changes in capillaries. Endoscopic acetic acid staining can produce a whitening effect on the surface of the gastric mucosa, so as to clearly present pit patterns, targetedly provide biopsy guidance and improve the diagnostic rate. Acetic acid staining combined with NBI was applied to this study, to investigate its value in the diagnosis of intestinal metaplasia in gastric mucosa.

\section{Data and methods}

\subsection{Research objects}

First-time patients, who were suspected to have atrophic gastritis and/or intestinal metaplasia under the observation of white light endoscope during normal gastroscopy performed from July of 2014 to July of 2015 in Endoscopy Center of Baogang Hospital, were chosen with informed consent forms signed. Patients with a previous history of gastric surgery, coagulation dysfunction, long-term use of non-steroidal anti-inflammatory drugs and other suspected malignant systemic lesions were excluded. A total of $72 \mathrm{pa}-$ tients were included in this research, including 40 males and 32 females, aged from 40 to 75 years, with the mean age of $(57.8 \pm 9.3)$ years.

\footnotetext{
*Correspondence: Meili Gao; E-mail: dcc66@ncspress.com; Address: Department of Gastroenterology, The Third Affiliated Hospital of Inner Mongolia Medical University, Baotou, Inner Mongolia, China.
} 


\subsection{Methods}

\subsubsection{Instruments and materials}

GIF-HQ290 Electronic Gastroscopy System with a NBI function (Olympus); Edible white vinegar (Laiyang Luhua Vinegar Food Co., Ltd.), which was made into $1.5 \%$ acetum in the preparation room of the hospital on the same day (white vinegar added by distilled water, adjusted $\mathrm{pH}=2.2$ ); Dyclonine hydrochloride mucilage (Yangtze River Pharmaceutical Group Co., Ltd., Batch No.: 15070421).

\subsubsection{Examination methods}

Gastroscopy was performed by the same endoscopic physician. Patients routinely took dyclonine hydrochloride mucilage before operation. Firstly, suspected lesions were rinsed with clean water repeatedly under the observation of the normal white light endoscope, with photographs taken. Then, switched to NBI mode to observe these photographs, and inserted the spray tube through the biopsy channel. $20 \mathrm{~mL}$ of acetum was uniformly sprayed on mucosal lesions, which needed observing and photographing. Finally, biopsy was performed in the white-patch area under the en- doscope. It was also performed to suspected lesions in the area without white patches under the normal white light endoscope. Biopsy specimens were sent for histopathological examinations and diagnosed by senior pathologists.

\subsection{Statistical analysis}

SPSS 13.0 statistical software was applied to this research, and the categorical data were compared by means of $\chi^{2}$. The difference $p<.05$ was of statistical significance.

\section{Diagnosis results of intestinal metaplasia in gastric mucosa}

\subsection{Endoscopic manifestations of intestinal meta- plasia in gastric mucosa}

Under the normal endoscope, it was shown that the mucosa was rough with nodular changes; uneven mucosa staining was shown under the normal NBI with visible white patches; with the help of acetic acid staining combined with $\mathrm{NBI}$, it was mainly shown that white patches in the mucosa were in the localized and the diffused types (see Figure 1).
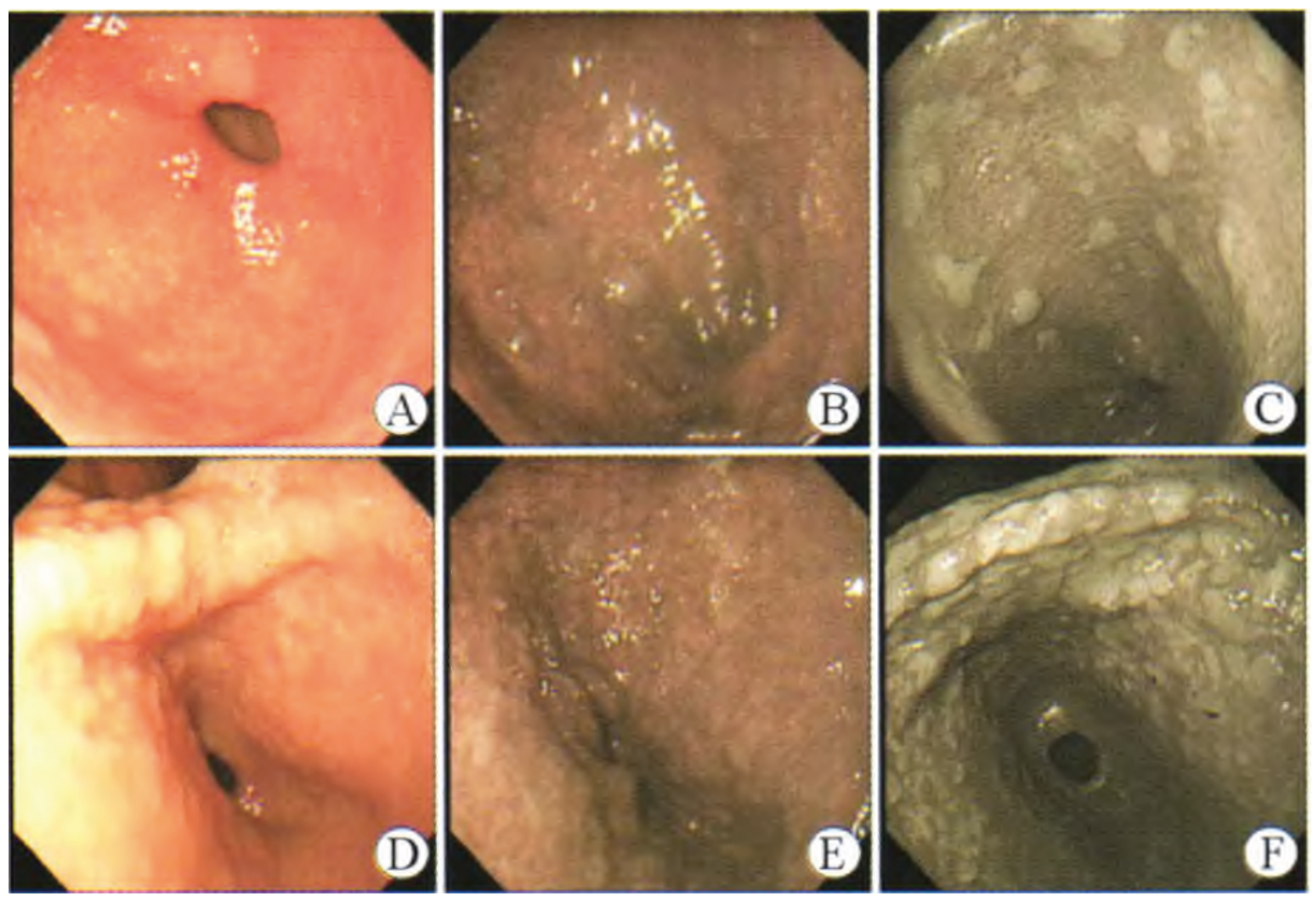

Figure 1: Flow chart of search results of studies from searching and screening

A. Mucosa in gastric antrum was rough and thinner, with diffuse erosion under the normal endoscope; B. Staining of mucosa in gastric antrum was uneven, with local white patches under the normal NBI; C Leukoplakia (white patches in mucosa) was observed in gastric antrum with the help of acetic acid staining combined with NBI; D. Mucosa in gastric angle was rough with nodular changes and visible white patches under the normal endoscope; E. Staining of mucosa in gastric angle was uneven with visible white patches under the normal NBI; F. Leukoplakia (white patches in mucosa) was observed in gastric angle with the help of acetic acid staining combined with NBI 
2.2 Comparison in pathologic results between normal NBI and acetic acid staining combined with NBI for the diagnosis of intestinal metaplasia

In view of pathological results, the sensitivity of normal NBI for the diagnosis of intestinal metaplasia in gastric mucosa was $80.0 \%$, and the specificity was $83.3 \%$; the sensitivity of acetic acid staining combined with NBI for the diagnosis of intestinal metaplasia in gastric mucosa was $96.7 \%$, and the specificity was $100 \%$; the differences in sensitivity and specificity of the two groups were statistically significant $(p<.05$, see Table 1$)$.

Table 1: Comparison of normal NBI and acetic acid staining combined with NBI for the diagnosis of intestinal metaplasia in gastric mucosa

\begin{tabular}{lllll}
\hline Group & Results & Pathological Positive Rate/n (\%) & Pathological Negative Rate/n (\%) & Total/n \\
\hline \multirow{2}{*}{ Normal NBI } & Positive & $48(80.0)$ & $2(16.7)$ & 50 \\
Total & Negative & $12(20.0)$ & $10(83.3)$ & 22 \\
Acetic acid staining & Positive & 60 & 12 & 72 \\
combined with NBI & Negative & $2(3.2)$ & 0 & 60 \\
Total & & 62 & $10(100.0)^{*}$ & 12 \\
\hline
\end{tabular}

Note. In comparison with normal NBI, ${ }^{*} p<.05$

2.3 Coincidence rate of acetic acid staining combined with NBI for the diagnosis of intestinal metaplasia in gastric mucosa with the pathological results

Acetic acid staining combined with NBI for the diagnosis of intestinal metaplasia in gastric mucosa was applied with 72 cases detected, and the pathological results showed 60 cases. The coincidence rate was $83.3 \%$ (60/72).

\section{Discussion}

Gastric carcinoma is considered to be the second most malignant tumor in terms of morality. Advanced gastric carcinoma has characteristics of poorly therapeutic efficacy and high mortality. However, the 5-year survival rate of early gastric carcinoma after surgery may be higher than $90 \% .{ }^{[2]}$ Therefore, how to detect lesions early becomes the current research priority. Endoscopic physicians have been endeavoring to detect lesions timely and perform biopsy accurately under the gastroscope.

The pathogenesis pattern of gastric carcinoma proposed by Correa ${ }^{[3]}$ has been acknowledged internationally, namely, the pattern is chronic superficial gastritis - chronic atrophic gastritis - intestinal metaplasia - atypical hyperplasia - gastric carcinoma. Among the precancerous lesions of gastric carcinoma, early diagnosis of intestinal metaplasia in gastric mucosa seems particularly significant. ${ }^{[4]}$ The remarkable diagnostic method of intestinal metaplasia in gastric mucosa is the application of gastroscopy combined with biopsy. Additionally, intestinal metaplasia is focally distributed mostly, and the normal endoscopy lacks in specificity. Therefore, it is easily misdiagnosed by means of random biopsy. The application of NBI and chromoendoscopy can make up for the deficiency of normal endoscopy. Besides, it can not only present the exact morphological characteristics of the surface of gastric mucosa, but also expose hidden lesions to obtain accurate targeted biopsy specimens and improve the detection rate of early lesions. This method has been gradually applied to clinical practice in recent years, and it is a common method for the diagnosis of early gastric carcinoma.

NBI was invented by Japanese scholars in 1999. It can filter out broad-band spectra in red, yellow and green lights by use of light filter, and select narrow-band spectra at $415 \mathrm{~nm}$ and $540 \mathrm{~nm}$. The narrow-band spectra can make the microstructure of the mucosa surface more clearly than the traditional white light endoscopy, with the threedimensional effect much stronger. Acetic acid is a type of food. It is easy-to-use, safe and widely used in clinical practice. Guelrud et al. ${ }^{[5]}$ used acetic acid for spraying the mucosa under the endoscope, whitening phenomenon appeared on the surface of the mucosa, so that the observation of gastric mucosa would be more intuitive and stereoscopic; In addition, acetic acid can neutralize a small number of alkaline gastric mucus, to the extent that the microstructure of the mucosa would be displayed more clearly. The research results showed that 48 cases of intestinal metaplasia in gastric mucosa were diagnosed in the normal NBI mode, while 60 cases were diagnosed by use of acetic acid staining combined with NBI, the latter was higher in diagnostic sensitivity and specificity than the normal NBI mode. It indicated that acetic acid staining combined with NBI can more accurately detect lesions of intestinal metaplasia in gastric mucosa. In addition, some studies have reported that the consistency of the normal endoscopy with the pathological diagnosis for intestinal metaplasia was relatively low, only ranged from $38.9 \%$ to $41.8 \%{ }^{[6]}$ This research showed that the coincidence rate of acetic acid staining combined with 
NBI for the diagnosis of intestinal metaplasia in gastric mucosa with the pathological results reached up to $83.3 \%$. The reason may be that acetic acid sprayed on the surface of mucosa can produce a whitening effect, which makes glandular mucosa thickened, whitened and easy to be observed, so that the diagnosis positive rate of intestinal metaplasia in gastric mucosa will be improved accordingly. ${ }^{[7,8]}$

The application of acetic acid staining combined with indicarmine or methylthionine chloride to the observation of gastric mucosa lesions has been reported by previous literatures. In comparison with the above-mentioned research, the advantage of this study is that NBI is a self-installed function of GIF-HQ290 Gastroscopy System and easy to operate; White vinegar is a type of food, which is relatively easy to obtain, simple to prepare, and helpful to the clinic practice. Therefore, it is a good choice to utilize acetic acid staining combined with NBI to improve the detection rate of precancerous lesions in patients with mucosal abnormalities

\section{References}

[1] Liu BY, Wang Y, Lei YF, et al. Clinical application value of endoscopic NBI in the diagnosis of intestinal metaplasia in gastric mucosa. Digestive Disease and Endoscopy. 2008; 2: 64-66.

[2] Choi IJ, Lee JH, Kim YI, et al. Long-term outcome comparison of endoscopic resection and surgery in early gastric cancer meeting the absolute indication for endoscopic resection. Gastrointest Endosc. 2015; 81: 333-341, e1. PMid: 25281498. https://doi.org/10. $1016 / j$.gie.2014.07.047

[3] Correa P. A human model of gastric carcinogenesis. Cancer Res. 1988; 48: 3554-3560. PMid: 3288329.

[4] Correa P, Houghton J. Carcinogenesis of helicobacter pylori. Gastroenterology. 2007; 133: 659-672. PMid: 17681184. https:// doi.org/10.1053/j.gastro.2007.06.026 under the normal endoscopy.

In summary, acetic acid staining combined with NBI is of a higher sensitivity and specificity in the diagnosis of intestinal metaplasia in gastric mucosa, which is in good consistency with the pathological diagnosis. Compared with the normal NBI, it is more capable to detect lesions of intestinal metaplasia in gastric mucosa, improve the accuracy of targeted biopsy to intestinal metaplasia, and has an important significance in the early detection and treatment of tumors. In addition, it is simple to use NBI, white vinegar is easy to obtain with a low price, and acetic acid has no carcinogenic risk. This method is of good safety, economy and operability, and worthy of further clinical promotion.

\section{Conflicts of Interest Disclosure}

The authors have no conflicts of interest related to this article.

[5] Guelrud M, Herrera I, Essenfeld H, et al. Enhanced magnification endoscopy: a new technique to identify specialized intestinal metaplasia in Barrett's esophagus. Gastrointest Endosc. 2001; 53: 559565. PMid: 11323579. https://doi.org/10.1067/mge.2001. 114059

[6] Zhou LY, Lin SR, Jin Z, et al. Endoscopic diagnosis of intestinal metaplasia in gastric mucosa. Chinese Journal of Digestive Endoscopy. 2001; 18: 84-86.

[7] Dinis-Ribeiro M. Chromoendoscopy for early diagnosis of gastric cancer. Eru J Gastroenterol Hepatol. 2006; 18: 831-838. https : //doi.org/10.1097/00042737-200608000-00005

[8] Kawahara Y, Takenaka R, Okada H, et al. Novel chromoendoscopic method using acetic acid-indigocarmine mixture for diagnostic accuracy in delineating the margin of early gastric cancers. Dig Endosc. 2009; 21: 14-19. PMid: 19691795. https://doi.org/10. $1111 / \mathrm{j} .1443-1661.2008 .00824 . \mathrm{x}$ 